Article

\title{
Power Demand Forecasting Using Long Short-Term Memory (LSTM) Deep-Learning Model for Monitoring Energy Sustainability
}

\author{
Eunjeong Choi ${ }^{1}$, Soohwan Cho ${ }^{2}$ and Dong Keun Kim ${ }^{3, *}$ \\ 1 Department of Computer Science, Sangmyung University, Seoul 03016, Korea; jung829c@gmail.com \\ 2 Department of Electrical Engineering, Sangmyung University, Seoul 03016, Korea; shcho@smu.ac.kr \\ 3 Department of Intelligent Engineering Information for Human, Institute of Intelligent Informatics \\ Technology, Sangmyung University, Seoul 03016, Korea \\ * Correspondence: dkim@smu.ac.kr
}

Received: 2 January 2020; Accepted: 2 February 2020; Published: 4 February 2020

\begin{abstract}
The purpose of this study is to design a novel custom power demand forecasting algorithm based on the LSTM Deep-Learning method regarding the recent power demand patterns. We performed tests to verify the error rates of the forecasting module, and to confirm the sudden change of power patterns in the actual power demand monitoring system. We collected the power usage data in every five-minute resolution in a day from some groups of the residential, public offices, hospitals, and industrial factories buildings in one year. In order to grasp the external factors and to predict the power demand of each facility, a comparative experiment was conducted in three ways; short-term, long-term, seasonal forecasting experiments. The seasonal patterns of power demand usages were analyzed regarding the residential building. The overall error rates of power demand forecasting using the proposed LSTM module were reduced in terms of each facility. The predicted power demand data shows a certain pattern according to each facility. Especially, the forecasting difference of the residential seasonal forecasting pattern in summer and winter was very different from other seasons. It is possible to reduce unnecessary demand management costs by the designed accurate forecasting method.
\end{abstract}

Keywords: Short-term; seasonal forecasting; power demand forecasting; Deep-Learning; LSTM; smart grid; power usage patterns

\section{Introduction}

Power demand forecasting is an important part of the Smart Grid. The prospect of a smart grid business is once again being reexamined through the transformation of low-carbon energy due to rising oil prices and environmental problems and the renewable energy business [1,2]. Research is being processed to diversify new industries by trying to combine them in various fields, such as IT, in preparation for energy issues [2,3].

Generally, energy is efficiently managed by applying the Smart Grid system using hardware and software that reflect the latest technology, in order to promote economic benefit using energy as a facility unit. Some key features of the Smart Grid, including energy scheduling management, require facilities to predict power demand in the facility. The essential purpose of electric power demand volatility assessment is not only blackout but also economic profit [4-6], because it interacted with intelligent demand response, which monitors energy in real time and manages demand of energy [4]. Utilizing demand responses can bring economic benefits to the facility, which will allow the country to achieve additional benefits such as cost savings and environmental conservation. Also, energy 
efficiency is the most profitable way for society to ensure energy supply, so researches on ways to consume energy efficiently are being actively carried out. The forecasting energy usage to ensure adequate energy supply is closely related to the energy efficiency increasing methods $[7,8]$. Also, energy efficiency (EE) can help the countries achieve multiple objectives such as lowering the energy bill, reducing energy dependence, decreasing greenhouse gas (GHG) and non-GHG emission, while maintaining or increasing the level of economic activity as well as improving overall sustainability by raising the share of renewable energy [9]. For example, countries such as China and Austria have set energy intensity targets as a percentage reduction compared to a certain base year $[9,10]$. Accurate forecasting of energy demand can reduce energy waste and improve energy sustainability. Indeed, many attempts are currently being made to forecast such power demand.

In the case of using Support Vector Machine, which is the most similar and generalized study, it is difficult to analyze an energy usage dataset in a facility-customized manner. It is difficult to deduce only the past power usage data, because it cannot recognize the change of the specific time zone by using only the existing machine learning algorithms [11]. It is a way to increase the accuracy by predicting the volatility of power demand in every time zone [12]. Since it is needed at each process, including data collection, preprocessing, feature extraction, and so on, power demand forecasting systems consume a lot of time and efforts [13,14].

In this study, we conducted power demand forecasting for each facility based on deep learning. In addition, we proposed a power demand forecasting model, "LSTM+MIDAS", that can confirm the volatility of the unconformities in comparison with the actual usage. This deep-learning system based on power demand volatility is necessary, because each facility has its own usage patterns, and the power usage, detailed power usage, contract capacity, and self-generated capacity vary from each facility [15-17]. However, there was one pattern at each facility type. After analyzing the data by season, day, month, and hour, we found a pattern of volatility in power demand. To analyze complex time series data such as power usage, many algorithms in the field of machine learning are applied in combination with regression analysis using dependent variables $[18,19]$.

The purpose of this study is to propose a power demand forecasting method and provide high accuracy of the predicted data; the goal is to reduce the error rate by more than $30 \%$ from the other methodologies. In addition, we confirmed the feature of power demand patterns and proposed efficient power demand forecasting methods for each facility. The most important function used in the volatility assessment is the usage forecasting study. Most studies have been conducted to predict and compare power demand using the autoregressive distributed lag (ARDL) and mixed-data sampling (MIDAS) methods. Those methods are used to estimate the value by numerical calculation using the different data formats that affect power demand. Therefore, in the power demand forecasting area, those are very actively applied. This section shows how the two approaches are used in power demand forecasting.

\subsection{ARDL Approach Method}

One way of predicting power demand is the ARDL, one of the most widely used dynamic regression analyses to analyze time series data. The ARDL, widely used as a methodology of error correction and cointegration, is a method mainly used for inferring numerical values in the social and economic fields [5,19].

In power demand forecasting, the ARDL method assumes that the monthly power demand affects the demand for power several years ago and includes autoregressive orders. The heating degree day and the cooling degree day are also included in the past month's independent variables. The statistical significance level can be confirmed by using different models according to the usage.

$$
y_{t}=\mu+\sum_{i=1}^{A_{y}} \alpha_{i} y_{t-i}+\sum_{j=1}^{A_{x}} \beta_{j} x_{t-j}+u_{t}
$$


Equation (1) is commonly used as an $\operatorname{ARDL}\left(A_{y}, A_{x}\right)$ model with autoregression degree of the dependent and independent variables $A_{y}$ and $A_{x}$ to predict monthly data $\left(y_{t}\right)$ from weekly data $\left(x_{t}\right)$ for the $j$-th week of the $t$-month [7]. It is assumed that the month is fixed at 4 weeks: $j=1, \ldots, 4$ [7]. However, power demand forecasting is very sensitive to temperature and seasonal factors due to the characteristics of power usage, so the older the past data, the less influence on forecast data [20]. Therefore, different weights should be assigned to each historical data to predict more accurately [20]. If each week's data in Equation (1) is given a different weight, Equation (2) can be derived where the $w$ is a week.

$$
y_{t}=a+\sum_{i=1}^{A_{y}} \alpha_{i} y_{t-i}+\sum_{j=1}^{A_{x}} \sum_{w=1}^{4} \beta_{w, t-j} x_{w, t-j}+b_{t}
$$

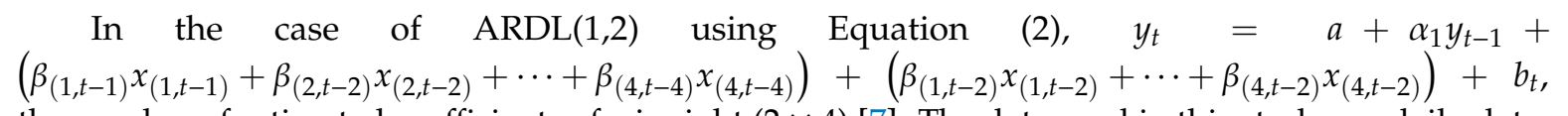
the number of estimated coefficients of $x$ is eight $(2 \times 4)$ [7]. The data used in this study are daily data, so the number of estimated coefficients is $60(2 \times 30)$, assuming 30 days per month. In this case, the estimation of the model itself becomes difficult and the reliability of the results is very low due to the degrees of freedom loss $[7,21]$.

\subsection{MIDAS Approach Method}

Like the ARDL method, the existing power demand forecasting system using the MIDAS method predicted power consumption with regression model [7], which is used to calculate GDP in economics. The biggest advantage of MIDAS is that the weight function automatically assigns weights [22].

$$
y_{t}=a+\sum_{i=1}^{D_{y}} \alpha_{i} y_{t-i}+\beta \sum_{j=1}^{D_{x} \times N_{w}} \varphi(j ; \theta) x_{w-j}+b_{t}
$$

The MIDAS Equation (3) is similar to the ARDL Equation (1) but includes a function $(\varphi(j ; \theta))$, which imposes different weights on high frequency (lags) [23]. $\theta$ is the parameter vector of the weight function, and $N_{w}$ is the number of weeks [7]. So, when predicting power demand by the MIDAS method, it is possible to predict by considering various external factors besides power demand data without adjustment to the parameters of the weight function. MIDAS regression is essentially tightly parameterized, reduced form regressions that involve processes sampled at different frequencies [24-26]. In order to assign different weights according to the frequency, the weight function used in the MIDAS method was used in this study. In other MIDAS regression forecasting studies, by setting the temperature, the number of working days, the income variable, and the price variable as independent variables, the accuracy of short-term power demand forecasting could have been improved [26,27]. Saturdays were set to be half days, excluding holidays and Sundays, and add up the number of workdays [7]. As a result, monthly data were useful in the power demand forecasting area. Relatively high accuracy was obtained when monthly and weekly heating and cooling differences and temperature, etc., were reflected. Also, as it is possible to analyze the pattern of volatility of power demand by separating weekday and weekend power demand data, forecasting can be made in facilities where there is a large difference in power demand between weekdays and weekends, such as a company and city hall. We focused on this feature in power demand and conducted a forecasting analysis. In the MIDAS method, various types of weight functions for calculating weights can be used, so the results can be different for each weight function. In short, the key idea of the model is to simplify the estimation of the weights imposed on the high frequency variables using only a few parameters.

The rest of the article is organized as five sections. Section 2 explains the data that is used in this study and method, Section 3 shows the results data, Section 4 shows the discussion points and, lastly Section 5 shows the conclusion. 


\section{Materials and Methods}

This section describes our dataset and LSTM power demand forecasting model as Figure 1.

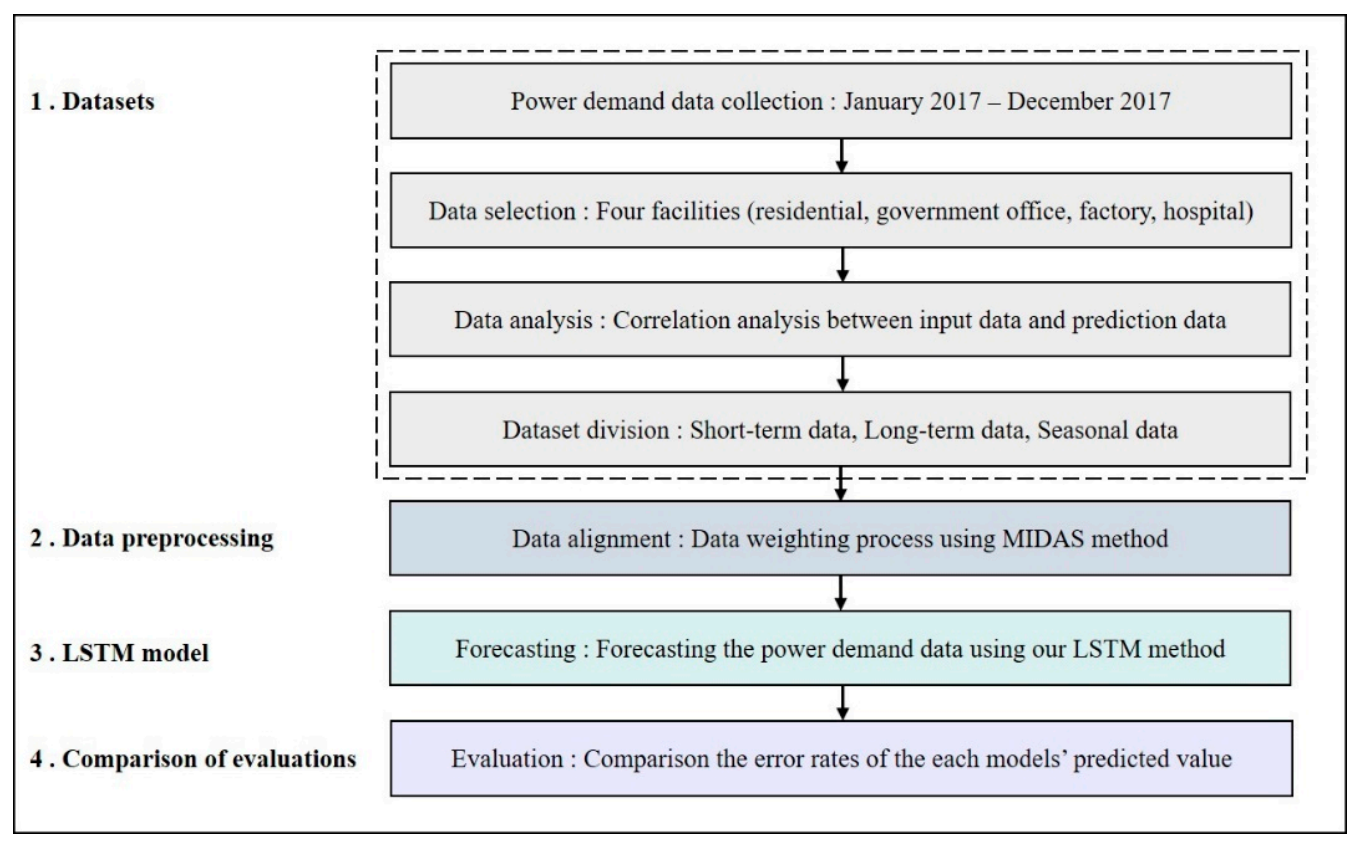

Figure 1. Framework structure of forecasting method.

From the results of previous studies of a volatility assessment model, we found that predicting power demand is the most important to understand the pattern of power demand data [28]. In order to identify the pattern, we classified the data into short-term and long-term data. Experiments were conducted through the collection of data for each facility according to power demand. We measured the three experiment methods' forecasting error rates to evaluate our forecasting model using data from different periods (short-term and long-term): (1) the existing power demand forecasting MIDAS algorithm; (2) the existing LSTM model; and (3) our proposed LSTM+MIDAS model. Using these three methodologies, we experimented with short-term data and long-term data to compare the differences in forecasting accuracy. However, in the case of residential facilities with large variable power demand patterns, it is important to understand the meaning from the seasonal, weather, and holiday aspects, rather than merely a period aspect. Then, our LSTM+MIDAS model performance was compared to other existing studies.

In Figure 2, in the case of residential and city hall buildings, the average maximum power demand tends to increase in summer (June to August), but the overall average maximum power demand of city hall was higher, and there was no difference between winter (November to January) and summer. Although there is a big difference in the power demand for each facility, the factory building showed the most similar average maximum power demand; between $600 \mathrm{kWh}$ and $700 \mathrm{kWh}$ over a year. Since hospital facilities are university hospital buildings, the maximum power demand was the highest among the four facilities. It also showed the highest maximum power demand pattern between May and September when the temperature rose. However, residential facility buildings showed the largest difference in the maximum power demand in summer and winter, while it showed similar patterns in other seasons, except summer. So, we conducted further experiments of seasonal power demand forecasting for residential facilities. 

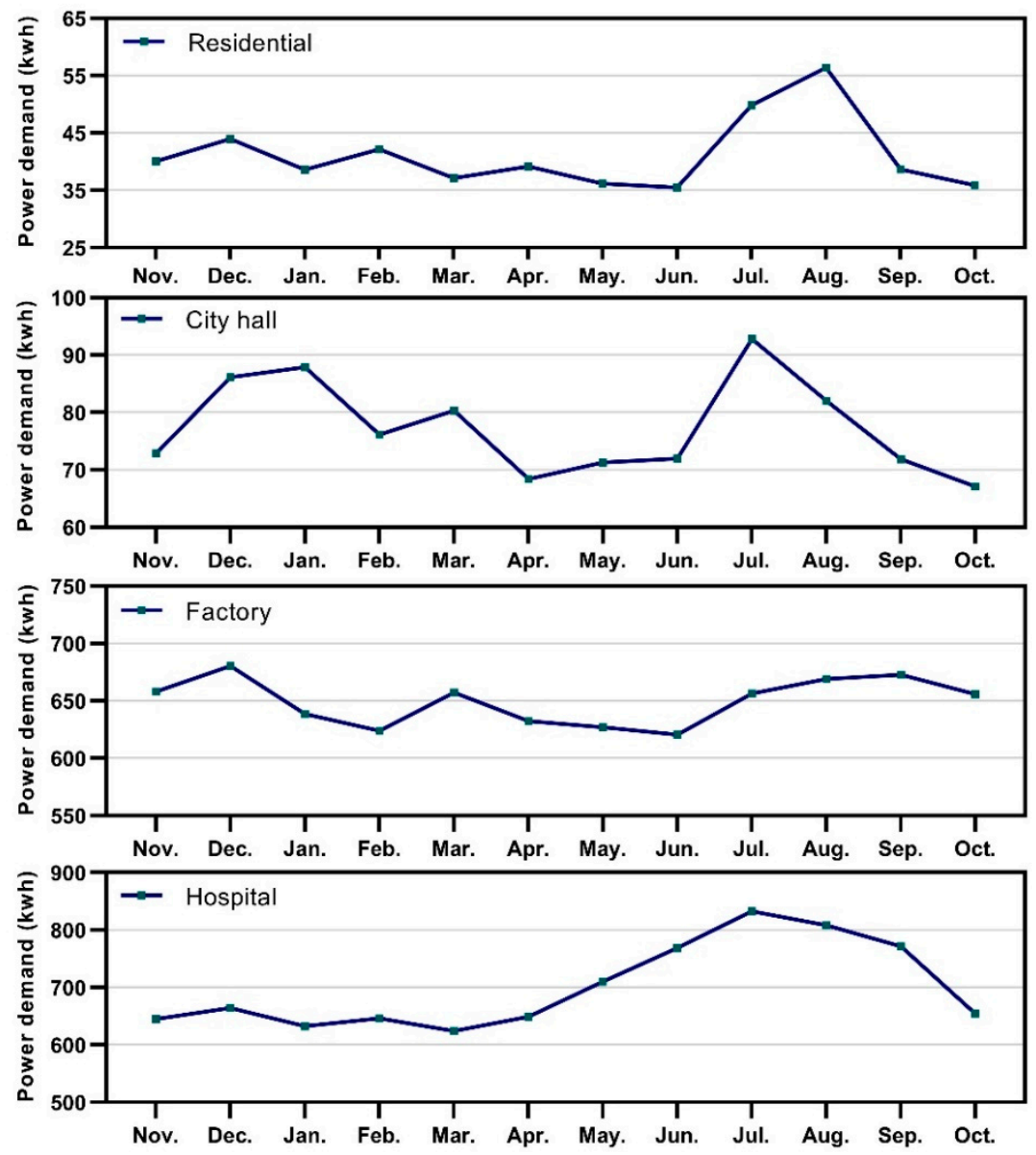

Figure 2. Average maximum power demand dataset for each facility's building.

\subsection{Datasets}

From November 2016 to October 2017, power demand was collected by sensors installed in various facility buildings (residential, hospital, farm, city hall, factory, company, etc.). Of these data, power demands from four institutions-residential, factory, hospital, and city hall-were used to calculate forecast accuracy for each power demand usage patterns. The collected data consist of 288 data per day every $5 \mathrm{~min}$, enabling detailed power pattern analysis, unlike other future forecasting power usage studies, which used quarterly power usage data [27]. For each of the four facilities, we used different data components as shown in Table 1 for short-term, long-term, and seasonal forecasts. In seasonal data, winter is classified from November 2016 to January 2017, and summer is from June to August 2017, based on Korean seasonal characteristics.

Table 1. The structure of the input and output datasets.

\begin{tabular}{ccccc}
\hline \multicolumn{2}{c}{ Data Component } & Short-Term Data & Long-Term Data & Seasonal Data \\
\hline \multirow{2}{*}{ Input } & Train data & 2 days $\times 288$ data & 8 days $\times 288$ data & 6 days $\times 288$ data \\
& Test data & 1 day $\times 288$ data & 4 days $\times 288$ data & 3 days $\times 288$ data \\
\hline Output & Forecasting data & 1 day $\times 288$ data & 4 days $\times 288$ data & 3 days $\times 288$ data \\
\hline
\end{tabular}

In the LSTM module, the train and test data of the input data are in a 2:1 ratio. Table 1 shows the structure of the input and output datasets. Since the power demand patterns are similar for each day of the week, input data (train data and test data) and output data consist of the same day (7-day lags) data. In the short-term forecast, we used three 7-day lags data in the previous three weeks to predict the next week's data for the same day of the week. In the long-term forecast, twelve 7-day lags data 
during the previous 12 weeks were used to predict data for the same day during the next four weeks. For example, every Monday, data during the previous 12 weeks was used as train and test data when the power demand data for every Monday was predicted during the next four weeks. Likewise, in seasonal data forecast, the previous nine weeks' 7-day lags data were used to predict the next three weeks. Figure 3 shows the flowchart of the proposed LSTM structure according to input and output data for each of the three models.

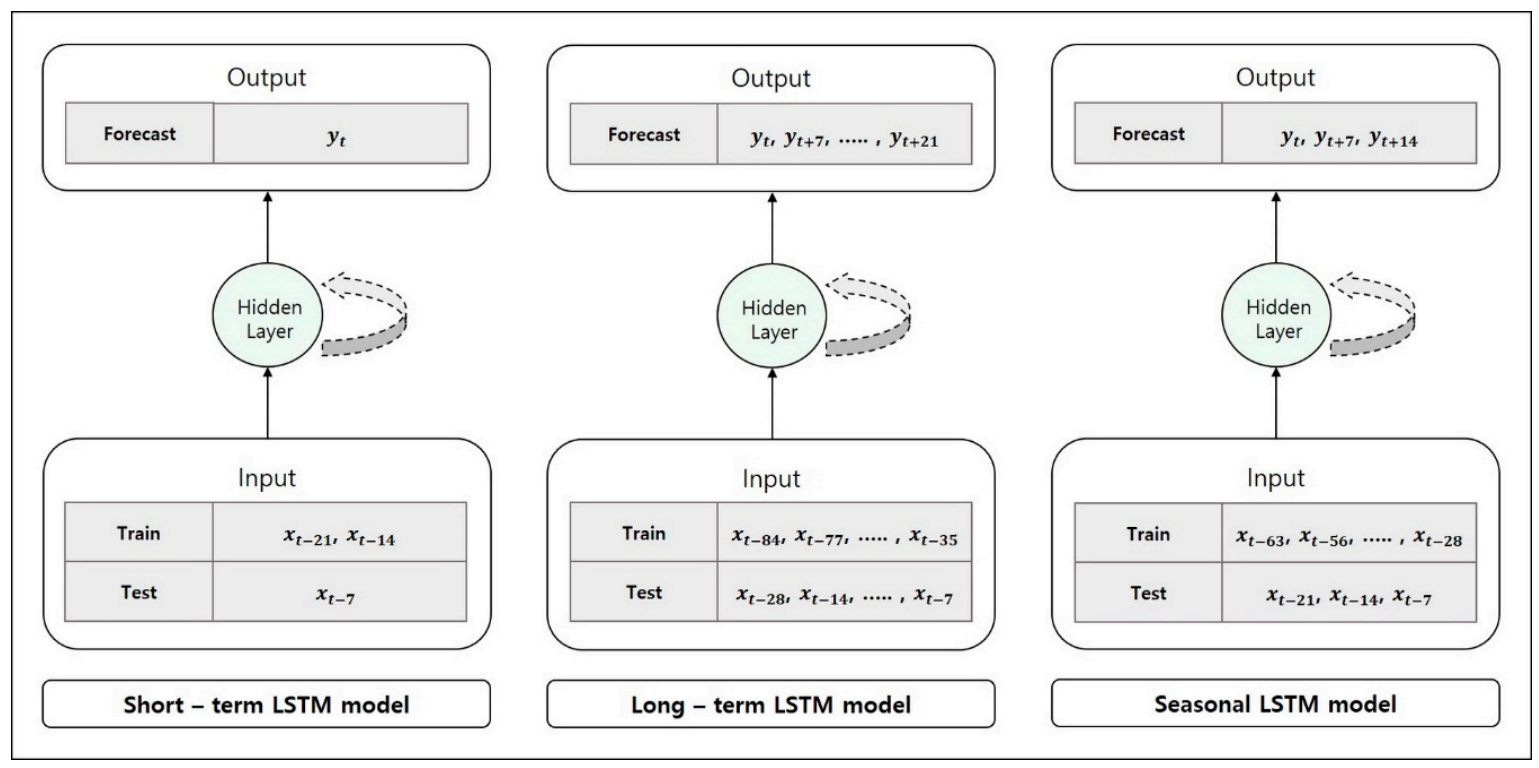

Figure 3. Proposed each LSTM model flowchart when the $t$ is the day.

\subsection{Data Preprocessing}

Before the previous power demand was entered as input data in the LSTM+MIDAS power demand forecasting model, we performed the preprocessing by assigning different weights to the input data affecting the volatility of the predicted data. Therefore, we conducted preprocessing using MIDAS method's weight function to weight each day's worth of input data, which is similar to the volatility of forecasting.

$x_{t}^{n}=\left\{x_{1}^{n}, x_{2}^{n}, x_{3}^{n}, \ldots, x_{t}^{n}\right\}$ is the input previous power demand, which means the power demand at $t$-th time of $n$th week's one day. In the MIDAS method, the weight value is calculated by using the MIDAS regression without directly estimating the weight given to the past value of the information variables. The function to determine the weight for high frequency values using the MIDAS regression method is:

$$
W(n ; \theta)=\frac{\exp \left(\theta_{1} n+\ldots+\theta_{t} n\right)}{\sum_{i=1}^{N} \exp \left(\theta_{1} n+\ldots+\theta_{t} n\right)}
$$

The results of the data preprocessing with the above Equation (4) are as follows:

$$
x_{t}^{\prime n}=W x_{t}^{n}+b
$$

Equation (4) is an ALMOD exponential function, which is widely used as a weighting function of the MIDAS regression method [29-31]. $\theta$ is the parameter vector of the weight function. The shape and speed of the weight function vary depending on the $\theta$ value [7]. We set the $\theta$ value in the range from -0.002 to 0.01 for exponential increase of the weight. $W$ is the vector matrix of $W(n ; \theta)$ and $b$ is a bias coefficient [32]. If the value after the calculation with the weight is too different from the raw data, it is adjusted through the adjustment of the bias value; the bias value is from -0.03 to 4.25. 


\subsection{LSTM-Based Power Demand Forecasting Model}

The LSTM's forecasting mechanism has been widely used for many time-series forecasting in recent years [33]. So, we used the LSTM model which is suitable for time-series forecasting, but we made it possible to reflect the weight function value used in the existing MIDAS to make the model, considering the power demand's volatility. Figure 4 shows the structure of the LSTM+MIDAS model for power demand used in this study.

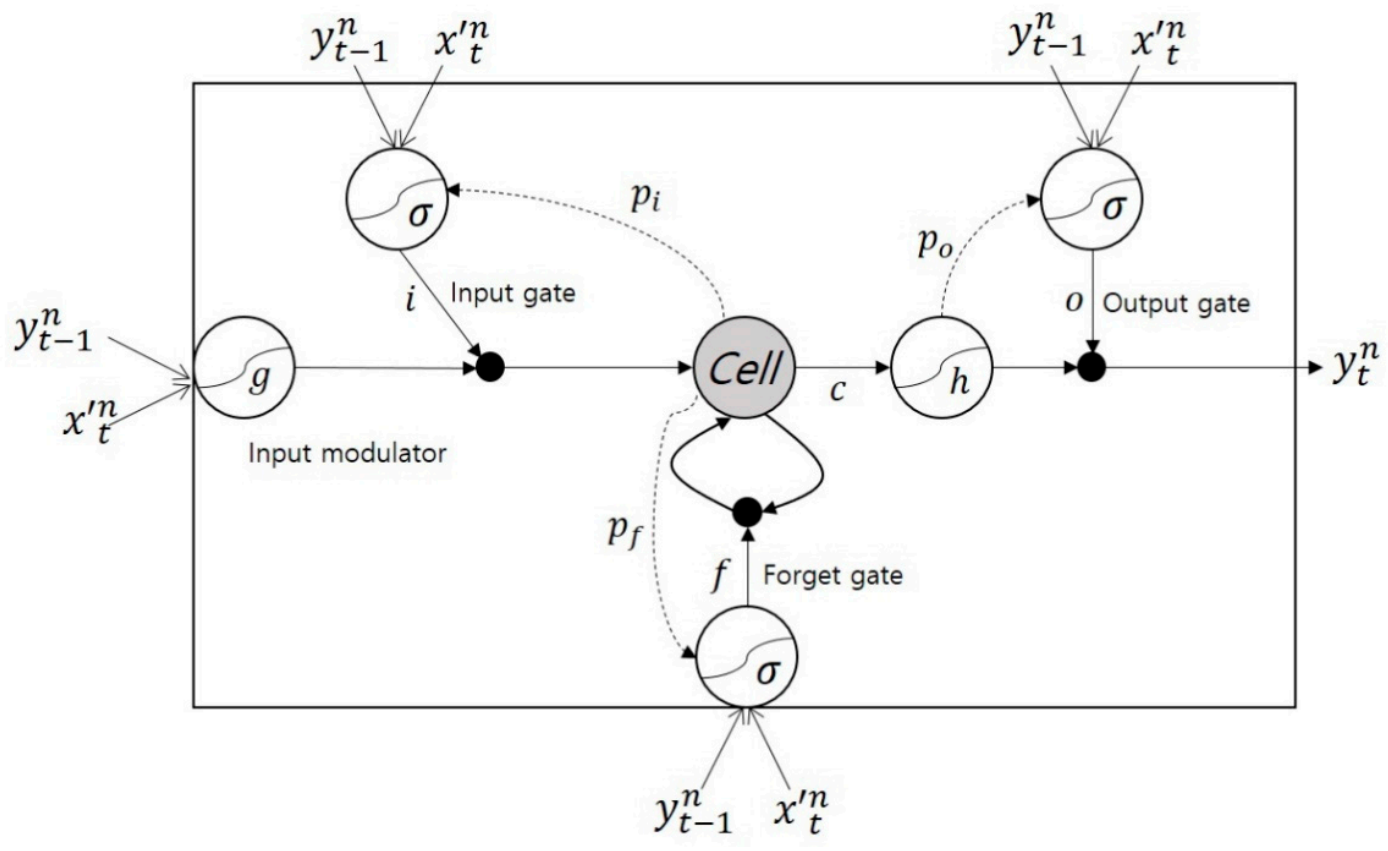

Figure 4. $L S T M+M I D A S$ model's structure.

In the LSTM+MIDAS model, the input data $x$ from the input layer are entered into the input gates, the forget gates, and the output gates as the parameters. The input parameters' values are entered by calculation of weight and bias value. At each gate, different values are calculated according to time $t$. The LSTM structure of the Figure 4 is operated as below:

$$
\begin{gathered}
z_{t}^{n}=g\left(W_{z} \boldsymbol{x}_{t}^{\prime n}+R_{z} y_{t-1}^{n}+b_{z}\right) \\
i_{t}^{n}=\sigma\left(W_{z} \boldsymbol{x}_{t}^{\prime n}+R_{z} y_{t-1}^{n}+p_{i} \odot c_{t-1}^{n}+b_{i}\right) \\
f_{t}^{n}=\sigma\left(W_{f} \boldsymbol{x}_{t}^{\prime n}+R_{f} y_{t-1}^{n}+p_{f} \odot c_{t-1}^{n}+b_{f}\right) \\
c_{t}^{n}=i_{t}^{n} \odot z_{t}^{n}+f^{t} \odot c_{t-1}^{n} \\
o_{t}^{n}=\sigma\left(W_{o} x_{t}^{\prime n}+R_{o} y_{t-1}^{n}+p_{o} \odot c_{t}^{n}+b_{o}\right) \\
y_{t}^{n}=o_{t}^{n} \odot h\left(c_{t}^{n}\right)
\end{gathered}
$$

In the above equations, $\boldsymbol{x}_{t}^{\prime n}$ and $\boldsymbol{y}_{t}^{n}$ represent the input and output at time $\mathrm{t} ; i_{t}^{n}, f_{t}^{n}$, and $o_{t}^{n}$ are the state values of the input gate, forget gate, and output gate; $c_{t}^{n}$ is the state value of the cell at time $t ; p_{i}, p_{f}$, and $p_{0}$-called peephole connections-are weight vectors connecting the internal memory value of the memory space and the gate; and $\odot$ indicates point-wise multiplication. $b_{i}, b_{f}$ and $b_{o}$ are bias values; the $\mathrm{W}$ is the input weight matrix; and $\mathrm{R}$ is the weight matrix for the input through the circulation. 


\subsection{Comparison of Evaluations for Each Model}

To evaluate the forecasting performance of LSTM model, we measured the statistical analysis using the mean relative error, mean absolute percentage error (MAPE), root mean square error (RMSE) and R-squared $\left(R^{2}\right)$. The formula for evaluating each model is as follows:

$$
\begin{gathered}
\text { MAPE }=\frac{100}{N} \sum_{i=1}^{N}\left|\frac{H_{i}^{*}-H_{i}}{H_{i}}\right| \\
\text { RMSE }=\sqrt{\frac{1}{N} \sum_{i=1}^{N}\left(H_{i}^{*}-H_{i}\right)^{2}} \\
R^{2}=1-\frac{\sum_{i=1}^{N}\left(H_{i}^{*}-H_{i}\right)^{2}}{\sum_{i=1}^{N}\left(\bar{H}-H_{i}\right)^{2}}
\end{gathered}
$$

where $H_{i}^{*}$ is the predicted values of the data $i, H_{i}$ is the actual values of the data $i$ and $\bar{H}$ is the mean of $H_{i}$. The normal range of the R-square is $[0,1]$, and the closer to 1 , the stronger the explanatory power of the model [6]. Since the power demand data used in this study varies in scale depending on the facilities, the $R^{2}$ was calculated to compare the predicted results according to the facilities.

\subsection{Development Environment}

In this study, we used Deeplearning4J (DL4J) to construct a power demand forecasting model using LSTM, which is the one of the most appropriate deep learning-based time series data forecasting methods [34]. DL4J has a characteristic that it is easy to construct an environment that can use graphics processing unit (GPU). So, we proposed a LSTM model based on the DL4J method combined with MIDAS to optimize for power demand forecasting.

\section{Results}

\subsection{Hyperparameter Setting}

The Good performance of deep learning can be achieved by setting the appropriate hyperparameters. The optimal number of layers, nodes, iterations, and activation function, etc., must be set. Generally, it is necessary to find the most optimal hyperparameter setting according to the number or purpose of the data. We found the hyperparameter setting, which achieved optimal results through a total of 40 settings in the hyperparameter setting. We set the number of hidden layers to 3 , the number of nodes to 10 , the learning rate to 0.01 , and set the number of iterations to 180 . We used hyperbolic tangent (tanh) and stochastic gradient descent as the activation function and optimization algorithm of the LSTM layer, respectively. In the classification LSTM model, cross-entropy (CE) and sum of square errors (SSE) are used as cost functions for prediction using multiclass classification, but mean square error (MSE) is predominantly used for prediction using regression [35-37]. Therefore, we used MSE as a cost function to reduce the prediction error. Table 2 shows a total of three results showing the highest accuracy obtained in the experiments to find the optimized hyperparameters. According to each setting, different prediction results were shown, we used Setting 3, which had the highest accuracy. 
Table 2. The hyperparameter setting value of the LSTM+MIDAS model.

\begin{tabular}{cccc}
\hline Hyperparameter & Setting1 & Setting2 & Setting3 \\
\hline Hidden layer & 2 & 3 & 3 \\
\hline The number of nodes & 10 & 8 & 10 \\
\hline Learning rate & 0.001 & 0.01 & 0.01 \\
\hline The number of iterations & 180 & 180 & 180 \\
\hline Activation function & Softmax & Hyperbolic tangent & Hyperbolic tangent \\
\hline Optimization algorithm & $\begin{array}{c}\text { Stochastic gradient } \\
\text { descent } \\
\text { LSE }\end{array}$ & $\begin{array}{c}\text { Stochastic gradient } \\
\text { descent } \\
\text { MSE }\end{array}$ & $\begin{array}{c}\text { Stochastic gradient } \\
\text { descent } \\
\text { MSE }\end{array}$ \\
\hline
\end{tabular}

\subsection{Comparison of Experimental Results}

Tables $3-5$ show the statistical analysis for the experiments conducted in this study by each facility building. Table 3 is the result of the short-term forecasting and Table 4 is the result of the long-term forecasting. In the case of the short-term forecasting, the MAPE of residential, city hall, factory, hospital facilities decreased from $21.04 \%$ to $10.44 \%$, from $15.6 \%$ to $2.73 \%$, from $7.21 \%$ to $1.63 \%$, and from $7.1 \%$ to $1.96 \%$, respectively. Table 4 shows the long-term forecasting results. There was no significant difference in each error rate by methodology.

Table 3. Error rates of the short-term forecasting results.

\begin{tabular}{cccccc}
\hline Model & Index & Residential & City Hall & Factory & Hospital \\
\hline \multirow{3}{*}{ MIDAS } & MAPE (\%) & 21.040 & 15.600 & 7.210 & 7.100 \\
& RMSE & 7.940 & 20.070 & 46.890 & 30.930 \\
& $\mathrm{R}^{2}$ & 0.302 & 0.750 & 0.370 & 0.926 \\
\hline \multirow{2}{*}{ LSTM } & MAPE (\%) & 19.401 & 4.714 & 4.060 & 2.892 \\
& RMSE & 3.365 & 7.623 & 23.302 & 15.246 \\
& $\mathrm{R}^{2}$ & 0.712 & 0.959 & 0.830 & 0.977 \\
\hline \multirow{2}{*}{ LSTM+MIDAS } & MAPE (\%) & 10.440 & 2.730 & 1.630 & 1.960 \\
& RMSE & 1.720 & 7.190 & 12.510 & 15.570 \\
& $\mathrm{R}^{2}$ & 0.917 & 0.962 & 0.893 & 0.981 \\
\hline
\end{tabular}

Table 4. Error rates of the long-term forecasting results.

\begin{tabular}{cccccc}
\hline Model & Index & Residential & City Hall & Factory & Hospital \\
\hline \multirow{3}{*}{ MIDAS } & MAPE (\%) & 34.610 & 11.500 & 7.380 & 4.040 \\
& RMSE & 7.950 & 17.560 & 59.700 & 37.070 \\
& $\mathrm{R}^{2}$ & 0.416 & 0.700 & 0.231 & 0.891 \\
\hline \multirow{2}{*}{ LSTM } & MAPE (\%) & 32.594 & 9.830 & 7.710 & 4.080 \\
& RMSE & 8.020 & 17.040 & 62.130 & 38.100 \\
& $\mathrm{R}^{2}$ & 0.439 & 0.766 & 0.235 & 0.880 \\
\hline \multirow{2}{*}{ LSTM+MIDAS } & MAPE (\%) & 32.500 & 8.700 & 7.700 & 4.050 \\
& RMSE & 7.330 & 14.490 & 53.610 & 36.800 \\
& $\mathrm{R}^{2}$ & 0.585 & 0.752 & 0.248 & 0.895 \\
\hline
\end{tabular}


Table 5. Error rates of the residential facility's seasonal forecasting results.

\begin{tabular}{ccccccc}
\hline \multirow{2}{*}{ Model } & \multicolumn{2}{c}{ MAPE (\%) } & \multicolumn{2}{c}{ RMSE } & \multicolumn{2}{c}{$\mathbf{R}^{\mathbf{2}}$} \\
\cline { 2 - 6 } & Winter & Summer & Winter & Summer & Winter & Summer \\
\hline MIDAS & 16.050 & 10.221 & 4.632 & 4.358 & 0.815 & 0.729 \\
LSTM & 16.200 & 6.520 & 12.628 & 2.830 & 0.094 & 0.886 \\
LSTM+MIDAS & 12.279 & 5.400 & 4.400 & 2.740 & 0.857 & 0.896 \\
\hline
\end{tabular}

Table 5 shows the residential facility's seasonal forecasting results. Unlike the long-term and short-term forecasting results in Tables 4 and 5, it can be confirmed that the error rates of the large difference are shown according to the methodology in the case of the experiment of the seasonal forecasting. In the winter experiments, the predicted data included the holiday, which drastically reduced the power demand. So, in the MIDAS method, when weighting value was assigned, we experimented by giving bigger weight to other holiday data. However, in the LSTM method, weight is not assigned, and thus shows the lowest performance in LSTM as shown in Table 5.

\subsection{Statistical Results}

LSTM+MIDAS were compared with results from short-term, long-term, and seasonal experiments with three models (MIDAS, LSTM, LSTM+MIDAS) using a nonparametric statistical test called the Friedman test [38]. The usual form of the Friedman test uses rank instead of the original values, where the ranks are obtained by ranking the rows separately and independently from each other [39]. In Table 6, $\mathrm{N}$ is the number of our total MAPE results (short-term, long-term, and seasonal), Chi-squared is 8.6 , Degree of freedom (DF) is 2, and the p-value is 0.018 . Friedman's critical value $\alpha$ is set at a 0.05 significance level, which has been commonly used in significance level [40].

Table 6. Friedman test results of all forecasting MAPE results.

\begin{tabular}{cc}
\hline & Friedman Test \\
\hline $\mathrm{N}$ & 10 \\
Chi-squared & 8.6 \\
Degree of freedom (DF) & 2 \\
$p$-value & 0.018 \\
\hline
\end{tabular}

\section{Discussion}

In this study, we proposed LSTM+MIDAS, a short-term data optimized power demand forecasting model using only the previous power demand data. The accuracy of the power demand forecasting depends on the data preprocessing, weight function approach. In addition, it is important to set up a model that can closely follow the pattern of power demand volatility over time. In Table 4, we confirmed that the LSTM+MIDAS model is better reflected in the power demand volatility than the other two methods. As general industrial facilities show similar patterns of power demand every day, short-term power demand forecasting using our model was very efficient. On the other hand, facility buildings that tend to respond sensitively to weekend and weather influences, such as residential, should be seasonally classified and then applied to our LSTM+MIDAS model.

To sum up the experiment's results, we confirmed the lowest forecasting performance for residential facilities, whereas the factory and hospital facilities showed a high accuracy with a relatively low level of error rates. The LSTM+MIDAS model of short-term data showed a higher level of forecasting than the other two methodologies. Also, the results of the short-term data are relatively better than those of the long-term data. We confirmed that, in comparison with the largest MAPE in the case of short-term forecasting, our LSTM+MIDAS model shows that the residential facilities decreased by $10.44 \%$ p, city hall by $12.87 \%$ p, factories by $5.58 \%$ p, and hospitals by $5.14 \%$ p; in the case of long-term forecasting, residential facilities only decreased by $2.11 \% \mathrm{p}$ and city hall by $2.8 \% \mathrm{p}$. Long-term 
forecasting did not improve the error rates in power demand forecasting at factories and hospital facilities. Generally, the power demand forecasting using the short-term data more accurately reflects the volatility, thereby improving the accuracy of short-term forecasting. However, the prediction of long-term data is highly influenced by weather and external factors, so the accuracy of the methodology does not show much difference, even though the overall accuracy is higher due to the larger number of datasets. We confirmed that there was no improvement in accuracy according to the methodology in the long-term power demand forecasting. Because the power demand data is related to the volatility, the long-term power demand data is not well predicted at the time of the volatility trends.

We also confirmed that predictions of power demand in residential facilities that are sensitive to weather, seasonal, and holiday influences can achieve higher accuracy by seasonally categorizing and predicting the data considering the weights for special situations, as shown in Figure 5.
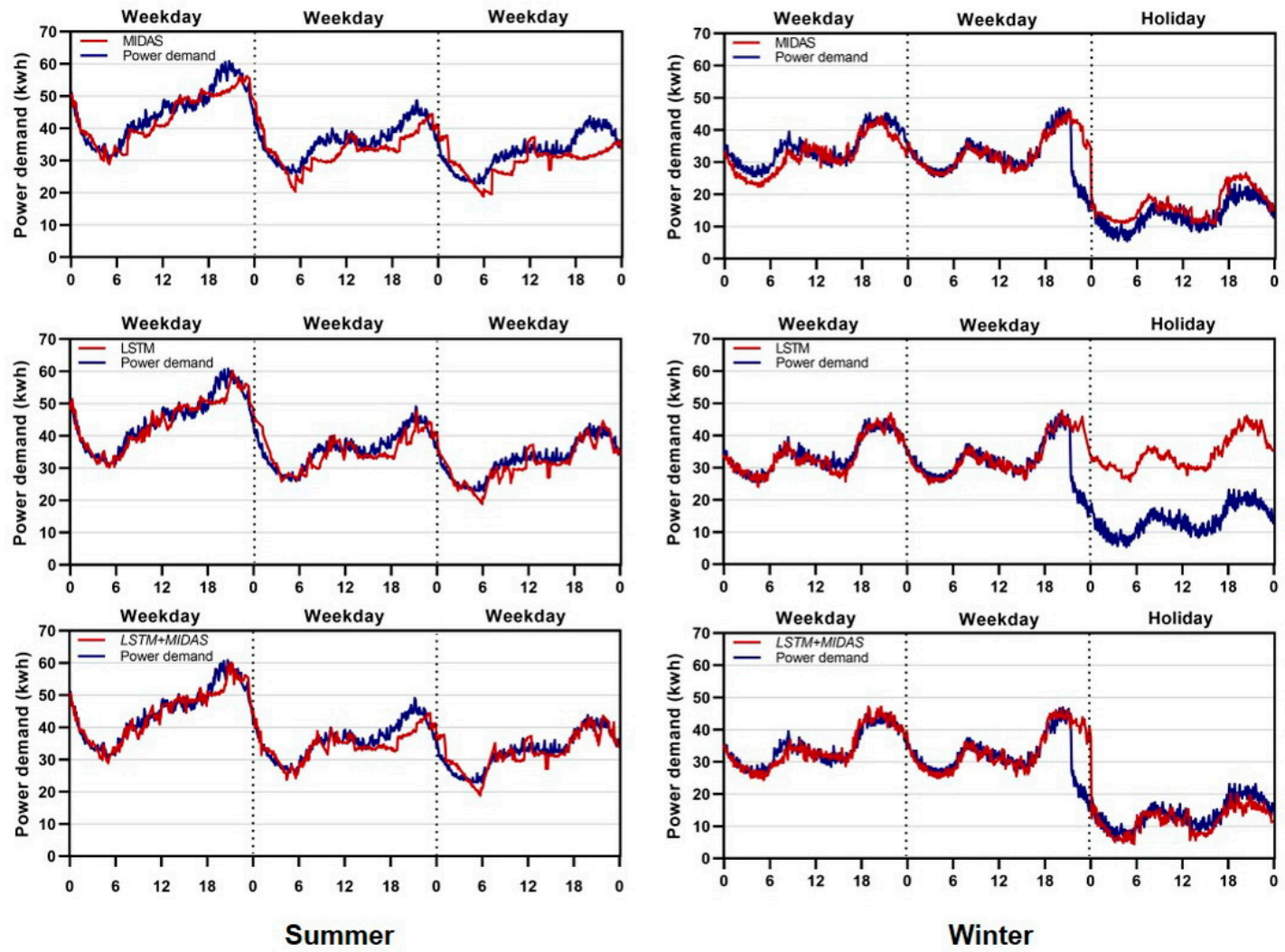

Figure 5. Residential seasonal power demand 3-day forecasting represented in 6-h intervals.

Other existing Cheng et al.'s study on power demand forecasting for residential facilities using PowerLSTM was used for July power demand input data [41]. We also used July data for summer forecasts, so we compared residential power demand forecasts for summer and Cheng et al.'s study [41]. The results show a comparison of the MAPE values of our model and Cheng et al.'s PowerLSTM model using residential data [41]. The MAPE of our $L S T M+M I D A S$ is $5.400 \%$, and the PowerLSTM is $8.935 \%$. The difference is $3.535 \%$ p and the error rate (MAPE) of LSTM+MIDAS is reduced by $39.564 \%$ compared to PowerLSTM [41]. In addition, we conducted the Friedman test to show whether the LSTM+MIDAS model is more meaningful than the MIDAS or LSTM model for the values of each experiment. We identified that the LSTM+MIDAS model is less than the $\alpha(p<0.05)$. So, we can say that the $L S T M+M I D A S$ model results are statistically significant through the Friedman test.

\section{Conclusions}

The existing power demand forecasting method using the regression analysis method required many external factors, in addition to the previous power demand data. However, in this study, 
it is possible to predict the power demand with reduced error rates only by the previous power demand data using the prediction of the patterns' trends. We could analyze that predicted power demand patterns were different depending on the facilities. Especially, residential facilities are very influenced by the seasonal factors. Since we considered only power demand data as input data, the forecasting error rate of residential facilities, which is affected by the weather, increased more than other facilities. The analysis of other factors affecting power demand can be added to predict power demand performances.

So, this study can be expected to further future studies, provide efficient and accurate forecasting of power demand by adding data on external factors affecting power demand predicting, as well as previous power demand data. In addition, predicting accurate power demand with high performance would be contributed to the sustainable development of the natural environment and environment management area, which are nowadays great issues all over the world.

Author Contributions: Writing—original draft preparation, E.C.; writing—review and editing, E.C. and D.K.K.; data curation, S.C. All authors have read and agreed to the published version of the manuscript.

Funding: This research received no external funding.

Acknowledgments: This work was supported by the Korea Institute of Energy Technology Evaluation and Planning(KETEP) and the Ministry of Trade, Industry \& Energy(MOTIE) of the Republic of Korea (No. 2018201060010C).

Conflicts of Interest: The authors declare no conflict of interest.

\section{References}

1. Jang, B.J.; Han, S.G. Energy-IT Fusion Technology Trends and Major Issues. JOK 2010, 28, 44-51.

2. Yang, E.S.; Kim, A.R.; Kim, B.A.; Shin, B.R. World Energy Outlook (WEO-2017) and Changes in Energy Demand and Supply. 2017. Available online: http://www.keei.re.kr/keei/download/WEIS1703.pdf (accessed on 4 December 2019).

3. Sung, M.J.; Shin, K.W. A Small-area Hardware Implementation of EGML-based Moving Object Detection Processor. JKIICE 2017, 21, 2213-2220.

4. Eum, J.Y. A Study on the Development of Energy Supply and Demand Forecasting Models for Smart City Energy Management System (CEMS). Master's Thesis, Sangmyung University, Seoul, Korea, 2015.

5. Zhang, B.; Pu, Y.; Wang, Y.; Li, J. Forecasting hotel accommodation demand based on LSTM model incorporating internet search index. Sustainability 2019, 11, 4708. [CrossRef]

6. Luo, T. Research on Decision-Making of Complex Venture Capital Based on Financial Big Data Platform. Complexity 2018, 2018, 5170281. [CrossRef]

7. Kim, C.H. Power Demand Forecasting Model Using Mixed Cycle Data. Research Report. 2014. Available online: http://www.keei.re.kr/web_keei/d_results.nsf/0/6632BB5F39AB536C49257E0D001A2348/\$file/\%EA\% B8\%B0\%EB\%B3\%B8\%202014-06\%20\%ED\%98\%BC\%ED\%95\%A9\%EC\%A3\%BC\%EA\%B8\%B0\%20\%EC\% 9E\%90\%EB\%A3\%8C\%EB\%A5\%BC\%20\%EC\%9D\%B4\%EC\%9A\%A9\%ED\%95\%9C\%20\%EC\%A0\%84\%EB\%

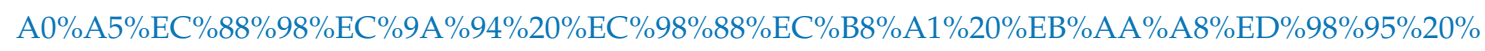
EA\%B5\%AC\%EC\%B6\%95.pdf (accessed on 10 December 2019).

8. Majd, M.; Safabakhsh, R. Correlational Convolutional LSTM for Human Action Recognition. Neurocomputing. In Press. Available online: https://www.sciencedirect.com/science/article/abs/pii/S0925231219304436 (accessed on 10 December 2019).

9. Bhadbhade, N.; Yilmaz, S.; Zuberi, S.J.; Eichhammer, W. The evolution of energy efficiency in Switzerland in the period 2000-2016. Energy 2020, 191, 116526. [CrossRef]

10. IEA. Insights Brief: Energy Efficiency Targets. Available online: https://webstore.iea.org/insights-briefenergy-efficiency-targets (accessed on 28 January 2020).

11. Jo, N.H. SVM Load Forecasting using Cross-Validation. Trans. Korean. Inst. Elect. Eng. 2006, 55A, 485-491.

12. Ishikawa, S.; Iwabuchi, K.; Takano, J. Peak power demand leveling to stabilize and reduce the power demand of dairy barn. Eng. Agric. Environ. Food 2016, 9, 56-63. [CrossRef]

13. Tang, R.; Li, H.; Wang, S. A game theory-based decentralized control strategy for power demand management of building cluster using thermal mass and energy storage. Appl. Energy 2019, 242, 809-820. [CrossRef] 
14. Rivera-González, L.; Bolonio, D.; Mazadiego, L.F.; Valencia-Chapi, R. Long-Term Electricity Supply and Demand Forecast (2018-2040): A LEAP Model Application towards a Sustainable Power Generation System in Ecuador. Sustainability 2019, 11, 5316. [CrossRef]

15. Lee, J.Y.; Kolasani, L. Security Based Network for Health Care System. APJCRI 2015, 1, 1-6. [CrossRef]

16. Law, R.; Li, G.; Fong, D.K.C.; Han, X. Tourism Demand Forecasting: A Deep Learning Approach. Ann. Tour. Res. 2019, 75, 410-423. [CrossRef]

17. Xu, P.; Du, R.; Zhang, Z. Predicting Pipeline Leakage in Petrochemical System through GAN and LSTM. Knowl. Based Syst. 2019, 175, 50-61. [CrossRef]

18. Petersen, N.C.; Rodrigues, F.; Pereira, F.C. Multi-output bus travel time forecasting with convolutional LSTM neural network. Expert Syst. Appl. 2019, 120, 426-435. [CrossRef]

19. Mohsen, B.O.; Ferda, H.; Scott, W.H. Mexican Bilateral Trade and the J-curve: An Application of the Nonlinear ARDL Model. Econ. Anal. Policy 2016, 50, 23-40.

20. Fousekis, P.; Katrakilidis, C.; Trachanas, E. Vertical Price Transmission in the US Beef Sector: Evidence from the Nonlinear ARDL Model. Econ. Model 2016, 52, 499-506. [CrossRef]

21. Li, T. A $(3,2)$ reduced degree-of-freedom unified zigzag laminated beam theory. Appl. Math. Model. 2020, 77, 1474-1496. [CrossRef]

22. Won, H.N.; Choi, K.W.; Choi, B.J. Forecasting GDP with a Mixed Data Sampling Model. IEJ 2016, $22,83-117$.

23. Mei, D.; Ma, F.; Liao, Y.; Wang, L. Geopolitical risk uncertainty and oil future volatility: Evidence from MIDAS models. Energy Econ. 2020, 86, 104624. [CrossRef]

24. Ghysels, E.; Qian, H. Estimating MIDAS Regressions via OLS with Polynomial Parameter Profiling. Econ. Stat. 2019, 9, 1-16. [CrossRef]

25. Andreou, E. On the Use of High Frequency Measures of Volatility in MIDAS Regressions. J. Econ. 2016, 193, 367-389. [CrossRef]

26. Zhou, Z.; Fu, Z.; Jiang, Y.; Zeng, X.; Lin, L. Can Economic Policy Uncertainty Predict Exchange Rate Volatility? New Evidence from the GARCH-MIDAS Model. Finance Res. Lett. In press. Available online: https://www.sciencedirect.com/science/article/abs/pii/S1544612319304982 (accessed on 28 January 2020).

27. He, Y.; Lin, B. Forecasting China's total energy demand and its structure using ADL-MIDAS model. Energy 2018, 151, 420-429. [CrossRef]

28. Seo, D.H.; Lyu, J.; Choi, E.J.; Cho, S.; Kim, D.K. Web based Customer Power Demand Variation Estimation System using LSTM. JKIICE 2018, 22, 587-594.

29. Ghyselsa, E.; Clarab, P.S.; Valkanovb, R. Predicting volatility: Getting the most out of return data sampled at different frequencies. J. Econ. 2006, 131, 59-95. [CrossRef]

30. Barndorff-Nielsen, O.E.; Corcuera, J.M.; Podolskij, M. Power variation for Gaussian processes with stationary increments. Stoch. Process. Their Appl. 2009, 119, 1845-1865. [CrossRef]

31. Barndorff-Nielsen, O.E.; Shephard, N. Power and bipower variation with stochastic volatility and jumps (with discussion). J. Financ. Econ. 2004, 2, 1-48.

32. Li, Y.; Han, C. Prediction for Tourism Flow based on LSTM Neural Network. Procedia Comput. Sci. 2018, 129, 277-283. [CrossRef]

33. Sideratos, G.; Ikonomopoulos, A.; Hatziargyriou, D.N. A novel fuzzy-based ensemble model for load forecasting using hybrid deep neural networks. Electr. Pow Syst. Res. 2020, 178, 106025. [CrossRef]

34. Eclipse Deeplearning4j. Available online: https://deeplearning4j.org/about (accessed on 11 December 2019).

35. Peimankar, A.; Sadasivan, P. An Ensemble of Deep Recurrent Neural Networks for P-wave Detection in Electrocardiogram. In Proceedings of the ICASSP 2019-2019 IEEE International Conference on Acoustics, Speech and Signal Processing (ICASSP), Brighton, UK, 12-17 May 2019; IEEE: Piscataway, NJ, USA, 2019. Available online: https://ieeexplore.ieee.org/document/8682307 (accessed on 4 February 2020).

36. Goki, S. Deep Learning from Scratch, 1st ed.; Hanbit Media: Seoul, Korea, 2017; pp. 111-112.

37. Goki, S. Deep Learning from Scratch 2, 1st ed.; Hanbit Media: Seoul, Korea, 2019; pp. 40-41.

38. Peimankar, A.; Weddell, S.J.; Jalal, T.; Lapthorn, A.C. Multi-objective ensemble forecasting with an application to power transformers. Appl. Soft Comput. 2018, 68, 233-248. [CrossRef]

39. Rohmel, J. The permutation distribution of the Friedman test. Comput. Stat. Data Anal. 1997, 26, 83-99. [CrossRef]

40. Demsar, J. Statistical comparisons of classifiers over multiple data sets. J. Mach. Learn. Res. 2006, 7, 1-30. 
41. Cheng, Y.; Xu, C.; Mashima, D.; Thing, L.L.V.; Wu, Y. PowerLSTM: Power Demand Forecasting Using Long Short-Term Memory Neural Network. In Advanced Data Mining and Applications: 13th International Conference, Singapore, 5-6 November 2017; Springer: Berlin, Germany, 2017; Available online: https://link.springer.com/ chapter/10.1007/978-3-319-69179-4_51 (accessed on 4 February 2020).

(c)

(C) 2020 by the authors. Licensee MDPI, Basel, Switzerland. This article is an open access article distributed under the terms and conditions of the Creative Commons Attribution (CC BY) license (http://creativecommons.org/licenses/by/4.0/). 